\title{
ARTICLE
}

Clinical Study

\section{A randomised controlled trial of an advance care planning intervention for patients with incurable cancer}

\author{
Stephanie B. Johnson ${ }^{1}$, Phyllis N. Butow ${ }^{1}$, Melanie L. Bell ${ }^{2}$, Karen Detering ${ }^{3}$, Josephine M. Clayton ${ }^{4}$, William Silvester ${ }^{5}$, Belinda E. Kiely ${ }^{6}$, \\ Stephen Clarke ${ }^{7}$, Lisa Vaccaro ${ }^{1}$, Martin R. Stockler ${ }^{6}$, Phillip Beale $^{8}$, Natalie Fitzgerald ${ }^{3}$ and Martin H. N. Tattersall ${ }^{1}$
}

\begin{abstract}
BACKGROUND: We modified and evaluated an advance care planning (ACP) intervention, which had been shown to improve compliance with patient's end of life (EoL) wishes, in a different patient population.

METHODS: Patients with incurable cancer, and a Family Member (FM), were randomised one-to-one to usual care or usual care plus an ACP intervention, between April 2014 and January 2017. Oncologists and participants were non-blinded. ACP was based on the Respecting Patient Choices model, with an offer to provide individualised ranges for typical, best-case and worst-case scenarios for survival time. Seven facilitators (two oncology nurses, two nurses and three allied health professionals) delivered the intervention within 2 weeks of study enrolment. The primary outcome measure, assessed by interviewing the FM 3 months after patient death, was the FM perception that the patient's wishes were discussed, and met.

RESULTS: Six hundred and sixty-five patients from seven Australian metropolitan oncology centres were referred for consideration by their oncologists, $444(67 \%)$ met the study inclusion criteria and were approached by a study researcher. Two hundred and eight patients (47\%) and their FM entered the trial as dyads. Fifty-three (46\%) dyads in the ACP group and 63 (54\%) dyads in the usualcare group had complete primary outcome data $(p=0.16)$. Seventy-nine patients and 53 FMs attended an ACP discussion. Mean length of discussion was $57 \mathrm{~min}$. FMs from 23 (43\%) dyads allocated to ACP and 21 (33\%) dyads allocated usual care reported the patient's EoL wishes were discussed and met (difference 10\%,95\% Cl: -2 to $8, p=0.27$ ). There were no differences in EoL care received, patient satisfaction with care; FM satisfaction with care or with death; or FM well being. Rates of palliative care referral were high in both groups (97\% vs $96 \%)$.
\end{abstract}

CONCLUSIONS: A formal ACP intervention did not increase the likelihood that EoL care was consistent with patients' preferences.

British Journal of Cancer (2018) 119:1182-1190; https://doi.org/10.1038/s41416-018-0303-7

\section{INTRODUCTION}

Professional bodies, physicians and researchers have voiced concerns about the quality of care for cancer patients nearing death. ${ }^{1,2}$ Decreasing aggressive interventions, respecting patient preferences for care and meeting patients' and families' psychosocial needs are increasingly important targets of optimal cancer care. $^{3,4}$ Advance care planning (ACP), a process that supports adults at any age or stage of health in understanding and sharing their personal values, life goals and preferences regarding future medical care, aims to ensure that people receive care that is consistent with these. ${ }^{5} \mathrm{ACP}$ is being promoted in many developed countries as a central component of End of Life (EoL) care. Although, existing research suggests that cancer patients infrequently engage in EoL discussions. ${ }^{6}$

Large and well-designed studies in EoL care are both challenging and uncommon. ${ }^{7}$ ACP for cancer patients was found to be associated with improved quality of life, reduced use of aggressive treatments at the EoL, and increased length of hospice stays in one North American study. ${ }^{8}$ In another American study, Mack et al. ${ }^{9}$ determined that patients with cancer are more likely to receive EoL care consistent with their preferences when they have had the opportunity to discuss their wishes for EoL care with a physician. Results of non-cancer studies evaluating ACP suggest that coordinated ACP interventions may result in improvements in concordance between patients' preferences for EoL care and the EoL care received ( 3 of 4 studies) ${ }^{10,11}$ However, with the majority of research to date conducted in the United States (US), and of low quality, $^{12,13}$ there is limited evidence to support the implementation of ACP interventions in different care settings and across cultures. $^{10}$

We report the results of a cancer-specific, multi-site Australian randomised controlled trial of an ACP intervention facilitated by a health-professional external to the treatment team. We hypothesised that ACP would: (a) increase discussion and documentation

\footnotetext{
${ }^{1}$ Centre for Medical Psychology and Evidence-based Decision-making (CeMPED), School of Psychology and Department of Medicine, University of Sydney, Sydney, NSW,

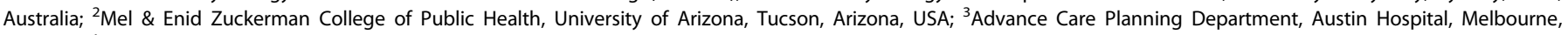

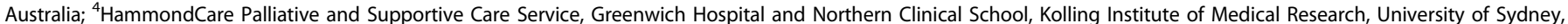

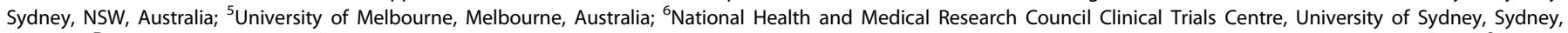

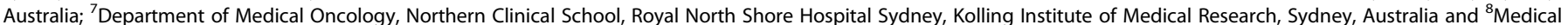
Oncology, Sydney Local Health District (SLHD), Royal Prince Alfred Hospital (RPA), Sydney, NSW, Australia Correspondence: Stephanie B. Johnson (Stephanie.johnson@sydney.edu.au)
} 
Table 1 Core components of the ACP intervention

I. Negotiate an agenda for the consultation

II. Assess the patient's and/or family's readiness to discuss future care

III. Establish the patients' preferred substitute decision maker(s)

IV. Explore the patients understanding of their medical situation, any unmet information needs and provide information if appropriate

V. Ask the patient for permission to discuss prognosis

If they wish to hear further information progress with providing best, worst and most likely scenarios.

VI. Explore the patient's values, goals, priorities, hopes, fears and concerns for the future

E.g. When you look at the future: what do you hope for? What worries you?

What is most important to you? What makes your life worth living?

If you were to become more unwell in the future:

What would be most important to you?

How would like to be cared for?

Is there anything else we should know about your wishes?

VII. Explore if there are any situations, treatments or health states the patient would find unacceptable

E.g. Is there anything that you worry about happening?

What is the worst medical outcome for you, that you still feel would give you quality of life?

Can you think of any circumstances where you would prefer the focus of treatment to be on comfort rather than extending life?

VIII. Summarise your understanding of the person's most important wishes for future care

IX. Consider any other specific treatment options relevant to the person's circumstances

Consider medical interventions such as: ICU admissions, invasive mechanical ventilation, non-invasive ventilation, IV fluids and antibiotics, chemotherapy (discuss treatment intent)

$\mathrm{X}$. Consider offering to make a recommendation for future medical care, if they were to become too sick to speak for themselves, based on their values and wishes

XI. Help the patient to document their wishes

of patient wishes for EoL care and increase perceived and actual compliance with patients' EoL wishes; (b) improve the quality of death; and (c) result in less mental and physical distress in family members.

\section{METHODS}

We conducted a prospective multi-site randomised controlled trial with two parallel groups receiving usual care plus a coordinated ACP intervention or usual care alone. The patient and a nominated family member/friend (hereafter, referred to as FM) were mailed questionnaires at baseline, and 6 weeks after completion of the ACP intervention, and then at 3 month intervals until the patient's death or study close (3 years after commencement). FM in this context included those closest to the person in knowledge, care and affection, including family, family-in-law or friends. The FM was contacted 3 months after the patient's death to complete a final structured interview and questionnaire. Following the patient's death, their medical record was reviewed for documentation of EoL preferences and assessment of the care received in the final 2 weeks of life.

Participants

Eligibility criteria included: age $\geq 18$ years, diagnosis of incurable cancer, expected survival time of 3-12 months (as estimated by the treating oncologist), prior systemic anticancer therapy, and ability to complete questionnaires and have an ACP conversation in English. Patients were excluded if they had previously completed formal ACP. Participating patients were asked to nominate an adult FM to participate in the trial with them; those unable to do so were excluded from the trial.

Participants were recruited from outpatient and inpatient departments of seven metropolitan oncology centres across two Australian states (2 in Victoria and 5 in New South Wales (NSW)). Patients were informed about the study by their treating oncologist. If interested in participating, a member of the research team followed up with the patient and FM to provide greater detail about the study, and obtain written consent. Consenting participants completed the baseline study questionnaire and were then randomised to the ACP intervention or usual care.

Randomisation and blinding

Participants were randomised centrally, using an interactive voice response system at the National Health and Medical Research Council (NHMRC) Clinical Trials Centre. Randomisation was balanced for by site using a minimisation algorithm. Assessors were blinded to the allocated treatment group, noting that intervention ACD documents might appear in patients' medical records. Oncologists and patients were not blinded.

\section{ACP intervention}

The ACP intervention and protocol for the study has been previously published. ${ }^{14}$ Seven facilitators (two oncology nurses, two nurses and 3 allied health professionals) received intervention training followed by peer mentoring and shadowing in the clinical environment. Intervention training was based on the Respecting Patient Choices model with the addition of skills in EoL communication. Participants in the intervention arm were additionally offered provision of individualised ranges for typical, best-case and worst-case scenarios for survival time based on their oncologist's estimate of expected survival time. ${ }^{15}$ Core components of the intervention are outlined in Table 1. The intervention was delivered in a structured meeting between the patient, their FM and the ACP facilitator, conducted within 2 weeks of study enrolment. The ACP facilitator reviewed the patient's medical notes and met with the patient's oncologist prior to intervention delivery to discuss medical goals of care, appropriate treatment options and the patient's prognosis. The intervention was audiorecorded for subsequent analysis. Oncologists were asked to review and sign completed Advance Care Directives (ACD). 
Patients were instructed that should their goals and wishes change at any stage, they should contact their ACP nurse to arrange another meeting.

\section{Measures}

Patients completed measures at baseline, 8 weeks ( 6 weeks postintervention), then every 3 months until death or the end of the study. Nominated family or friends completed measures at baseline, 8 weeks, every 3 months until the patient's death and at 3 months after the patient's death. Following the patient's death, a review of their medical record assessed documentation of EoL preferences and medical interventions received in the final 2 weeks of life. Measures, and their timings, are presented elsewhere. $^{14}$

Primary outcome. The primary outcome of this trial was FM perception that the patients' EoL wishes were discussed, and complied with. This binary outcome was based on FM response 3 months after the patient's death to the questions: (1) "Did the patient discuss with you any particular wishes he/she had about the care they would want to receive if they were dying", (response options were on a five point Likert scale from $0=$ "Not at all" to 5 $=$ "Very much)"; and (2) "I am satisfied that at the end of his/her life their wishes were met" (response options were on a five point Likert scale from 0 "Strongly disagree" to $5=$ "Strongly agree"). This outcome was scored positive if the FM reported that EoL wishes were discussed ("Quite a bit" or "Very much") and if the patient's EoL wishes were met (responses of "Agree" or "Strongly agree"). All other responses were coded as negative.

The documentation of patient preferences for EoL care and concordance with care received at the EoL. The main secondary outcomes were concordance between documented patient preferences for EoL care and care received at the EoL, calculated for each of the following aspects of care: place of death (hospital, home, hospice/palliative care unit, other); medical interventions in the last 2 weeks of life (CPR, intensive care unit [ICU] admissions, life prolonging treatments, surgery, mechanical ventilation and 'other' significant interventions) and chemotherapy in the last 4 weeks of life. Care received was assessed by review of medical records (inpatient and outpatient) at the patient's cancer treatment hospital and affiliated palliative care unit(s). For patients who died at home it was assumed that aggressive interventions were not received. Concordance was rated positive either if: (a) patients expressed a preference for a specific intervention and received that intervention or (b) patients expressed a preference not to receive a specific intervention and did not receive that intervention.

Other secondary outcomes collected from record review included the prevalence, timing and location of EoL care documents and discussions, and receipt of aggressive treatments (hospital admission, ED admission, Intravenous Antibiotics, Dialysis). An ACD was defined as any formal document that sets out a person's wishes about future medical treatment if they lose their capacity, which had been personally signed by the patient. 'Medical orders' such as a Do Not Resuscitate (DNR) order, were not recorded as ACDs. We also recorded non-formal documentation-'other EoL documentation'-defined as evidence of discussion with the patient regarding (1) the patient's preferences for care or (2) that these could not be elicited. For example, evidence of discussion regarding patient goals of care or preferences regarding cessation of chemotherapy in the oncology clinic was recorded as 'other EoL documentation.' A health professional's recommendation for care or a 'medical order' were not deemed to constitute a 'patient preference'.

Data were collected using a standardised form and manualised coding protocol. Coders received three days of training and attended weekly meetings with the study coordinator to discuss and reach consensus on scoring. Disagreements between coders or unusual cases were discussed and referred to the whole research team for final consensus. Percentage of agreement (POA) was calculated for each item and overall. Cohen's Kappa was used to measure interrater reliability ${ }^{16}$. POA was above $90 \%$ for all items and overall, and Cohen's kappa agreement was $>0.7$ (excellent agreement) for all variables.

Quality of EoL care. Quality of EoL care was measured 3 months after the patient's death using a 27-item structured interview adapted from tools used by Detering et $\mathrm{al}^{12}$ and Engelberg et $\mathrm{al}^{17}$ assessing FM satisfaction with the quality of a patient's death. Response options were on 5 point Likert scales, with higher scores indicating greater satisfaction.

The impact of death on surviving family members. Anxiety, depression and quality of life (QOL) of FMs were assessed using the Hospital Anxiety and Depression Scale (HADS) ${ }^{18}$ and the SF12 ${ }^{19}$ measured at baseline, every 3 months until the patient's death, and 3 months after death. Scores on the HADS subscales range from 0 to 21 ; higher scores indicate worse mental health. Australian weights were used to score the SF-12; scores on mental and physical well-being subscales range from 0 to 100; higher scores indicate better QOL. The impact of death on surviving FMs was measured using the Impact-of-Events Scale (IES) ${ }^{20}$ at 3 months after death. Scores on the IES range from 0 to 75 ; higher scores indicate greater distress.

Patient-family and patient-healthcare provider communication about EoL care. Patients rated Patient/FM and Patient/healthcare provider communication about EoL care using items adapted from Wright et $\mathrm{al}^{8}$ at baseline and 6 weeks post-intervention using a 6-point Likert scale from 0 "Not all" to 5 "Very Much" discussion.

Patient and caregiver satisfaction with care. Patient and FM satisfaction with care was assessed at 6 weeks post-intervention using a 5 question survey utilised in a previous trial ${ }^{12}$ focusing on satisfaction with information provision. Scores range from 0 to 24; higher scores indicate higher satisfaction.

Patient and family satisfaction with the ACP intervention. Patient and FM satisfaction with the ACP intervention was assessed using a 9-item study-developed questionnaire; higher scores indicate higher satisfaction.

Intervention fidelity. Intervention fidelity was coded using a standardised form and coding protocol, by two reviewers. Possible scores for adherence to content, ranged from 0 to 11 .

\section{Sample size}

In a previous trial by the investigator group ${ }^{12}$ EoL wishes were known and respected in $86 \%$ of the intervention group compared to $30 \%$ of controls. Assuming the same baseline rate of EoL wishes known and respected in cancer patients, and believing a doubling to $60 \%$ would influence clinical practice, we estimated that two study groups that each include 56 patients who die within the 3 year follow up period would result in the study having $90 \%$ power to detect a between-group difference with $95 \%$ certainty. A conservative estimate of mortality was $75 \%$. To allow for incomplete data on $20 \%$ of patients and a further $10 \%$ of their FM, we proposed a sample size of 210 patients with incurable cancer.

\section{Statistical methods}

Cross-sectional categorical and continuous outcomes were tested using chi-square and $t$-tests, respectively, to compare the ACP and usual-care arms. We tested for the effect of nesting within oncologist using mixed models and including a random effect for 
Completed baseline assessment $(n=100)$

Withdrew $(n=1)$

Missed session $(n=3)$

Allocated to intervention $(n=104)$

Received allocated intervention $(n=79)$

Did not receive allocated intervention $(n=25)$

Patient withdrew $(n=9)$

Patient died $(n=9)$

Patient refused ACP $(n=5)$

Unable to schedule or contact $(n=2)$

Completed 6 weeks post intervention follow up assessment $(n=67)$

Withdrew $(n=16)$

Died before first follow up $(n=17)$

Missed session $(n=4)$

Family completed bereavement
interview $(n=53)$
Patient still alive at close out
$(n=20)$
Withdrew $(n=21)$
Missed session $(n=10)$

Analysed at medical record review $(n=67)$

Patient still alive $(n=20)$

Withdrew permission to collect further data $(n=9)$

Couldn't access records $(n=8)$
Excluded $(n=457)$

Not meeting inclusion criteria $(n=128)$

Incomplete referrals or unable to contact $(n=93)$

Declined to participate $(n=236)$

72 Not interested

41 Too unwell

17 Time issues

17 Too distressing

17 Trial dyad refused to participate

16 Unable to contact

13 Travel issues

2 No reason given

22 Other

19 Missing data

Fig. 1 CONSORT flow diagram

oncologist. These effects were estimated at 0 , and were therefore excluded. Concordance of patient preferences and actual care was tested with chi-square or Fisher's exact test (in the case of sparse data). Timing of EoL care documentation and palliative care were tested using the Wilcoxon Rank Sum test. Longitudinal outcomes, including FM anxiety and depression, physical and mental well being were estimated using linear mixed models. We estimated the difference between arms using contrasts within these models. We used logistic regression to investigate factors associated with the outcomes of EoL wishes discussed and met. Longitudinal outcomes, including family member anxiety and depression, physical and mental well being were estimated using linear mixed models. We estimated the difference between arms using contrasts within these models. The post-baseline, prebereavement means were estimated and compared. For postbereavement, the change from baseline to the bereavement interview were estimated. Stress, as measured by the impact-ofevents scale, was tested using a $t$-test, as it was only assessed at the bereavement interview.

We used logistic regression to investigate factors associated with the outcomes of EoL wishes discussed and met, and receipt of aggressive EoL care as defined by receipt of CPR; ventilation; admission to the ICU, admission to the ED, chemotherapy or surgery, in the last 2 weeks. These factors included the following patient factors: ACP intervention, patient age, gender, state (New South Wales, Victoria), marital status, medical training (yes/no), living alone, country of birth (Australia/not Australia), religion (yes/ no), health insurance (yes/no), survival time, CPR preference, advanced care directive (yes/no), substitute decision maker, advanced care planning evidence from medical record review, self-reported communication about EoL wishes (with doctor, family, other health providers; with palliative care doctor or nurse), chose to receive information on life expectancy and the patient's satisfaction with care total. Family characteristics tested were: age, gender marital status, medical training, education, living alone, country of birth, ethnicity, religion and health insurance.

Sensitivity analyses for primary outcome and missing data The primary outcome was whether EoL care wishes were discussed and met. As is common in EoL research, there was a high rate of missing data. We carried out a chi-square test to compare arms, on family members who participated in the bereavement interview, and had complete data on this outcome. This analysis makes a "missing completely at random" assumption, i.e., subjects analysed are no different from subjects who dropped out. If this is not true, estimates may be biased. ${ }^{2}$ Therefore, we undertook sensitivity analyses to assess the robustness of results to the assumptions made about the missing data, by assuming data were missing at random, i.e., by using multiple imputation conditioning on variables associated with the outcome, missingness, or both and performed multiple imputation under a "missing not at random" assumption, imputing missing values using the same variables as above, but from the usual-care group only (control group imputation). ${ }^{21}$ We also compared baseline 
Table 2 Demographic and clinical characteristics of participants and family members

\begin{tabular}{|c|c|c|}
\hline & $\mathrm{ACP}$ & Usual care \\
\hline \multicolumn{3}{|l|}{ Patient Characteristics } \\
\hline Age Mean (range) & $66(34-90)$ & $65(31-82)$ \\
\hline \multirow{2}{*}{$\begin{array}{l}\text { Months from study entry to death } \\
\text { Median (range) }\end{array}$} & $5.3(0.4-18.3)$ & $5.5(0.3-22.1)$ \\
\hline & $n(\%)$ & $n(\%)$ \\
\hline Gender (male) & $56(53.9)$ & $55(52.9)$ \\
\hline Married & $69(66.3)$ & $66(63.5)$ \\
\hline Lives alone & $20(19.3)$ & $11(10.6)$ \\
\hline Medical training (yes) & $13(12.5)$ & $13(12.5)$ \\
\hline \multicolumn{3}{|l|}{ Ethnicity } \\
\hline Australian-New Zealand & $72(69.2)$ & $74(71.2)$ \\
\hline European & $14(13.5)$ & $13(12.5)$ \\
\hline Other & $14(13.5)$ & $13(12.5)$ \\
\hline Private Health Insurance (yes) & $58(55.8)$ & $51(49.0)$ \\
\hline \multicolumn{3}{|l|}{ Cancer type } \\
\hline Lung & $30(28.9)$ & $28(26.9)$ \\
\hline Urological & $13(12.5)$ & $6(5.8)$ \\
\hline Pancreas & $12(11.5)$ & $9(8.7)$ \\
\hline Other Gl & $11(10.6)$ & $9(8.7)$ \\
\hline Gynaecological & $8(7.7)$ & $8(7.7)$ \\
\hline Breast & $7(6.7)$ & $6(5.8)$ \\
\hline Colorectal & $6(5.8)$ & $17(16.35)$ \\
\hline Other & $6(5.8)$ & $5(4.8)$ \\
\hline Missing & $4(3.9)$ & $3(2.9)$ \\
\hline Skin & $3(2.9)$ & $3(2.9)$ \\
\hline Head and neck & $2(1.9)$ & $2(1.9)$ \\
\hline Bone and soft tissue & $1(1.0)$ & $4(3.9)$ \\
\hline Unknown Primary & $1(1.0)$ & $3(2.9)$ \\
\hline CNS & $0(0.0)$ & $1(1.0)$ \\
\hline \multicolumn{3}{|l|}{ Family characteristics } \\
\hline \multirow[t]{2}{*}{ Age Mean (range) } & $58(21-84)$ & $58(20-83)$ \\
\hline & $n(\%)$ & $n(\%)$ \\
\hline Male & $23(22.1)$ & $32(30.8)$ \\
\hline Married & $84(80.8)$ & $76(73.1)$ \\
\hline Medical training & $16(15.4)$ & $17(16.4)$ \\
\hline \multicolumn{3}{|l|}{ Ethnicity } \\
\hline Australian-New Zealand & $78(75.0)$ & $77(74.0)$ \\
\hline European & $8(7.7)$ & $12(11.5)$ \\
\hline Other & $11(10.6)$ & $8(7.7)$ \\
\hline Private Health Insurance & $43(41.4)$ & $37(35.6)$ \\
\hline
\end{tabular}

characteristics of patients and FMs who had complete data on the primary outcome with those who did not.

\section{RESULTS}

Of the 665 patients referred by oncologists, 444 (67\%) met the study inclusion criteria and were approached by a study researcher between April 2014 and December 2016. Two hundred and eight patients (47\% of those eligible) and their nominated FMs entered the trial as dyads. (See Fig. 1: CONSORT flow diagram). Primary reasons for non-participation included 'not being interested' $(n=72)$ and feeling too unwell $(n=41)$. Fiftythree $(46 \%)$ dyads in the ACP group and 63 (54\%) dyads in the usual-care group had complete primary outcome data $(p=0.16)$. Patient data were collected between April 2014 and December 2016, and FM data between April 2014 and January 2017.

Baseline demographic and clinical variables were similar between the arms (Table 2). Patients were on average 66 years old, and FMs 58 years. Two thirds of participants were born in Australia. Genders were equally represented in patients; $73 \%$ of FMs were female. The most common cancer type was lung, followed by urological and pancreatic. Median survival of the patients was 5.4 months, with similar mortality rates in the two treatment groups (ACP $87 \%$ and usual care $84 \%$ ).

Advance care planning

Overall, 79 patients and 53 FMs attended an ACP discussion. Mean length of discussion was $57 \mathrm{~min}$ (range 18 to $120 \mathrm{~min}$ ). Eighteen (23\%) patients chose to receive individualised scenarios for expected survival time and five (6\%) patients requested a second ACP meeting. Patient and FM satisfaction with ACP was high: percentage agreeing or strongly agreeing ranged from $69 \%$ to $91 \%$ for the 9 patient satisfaction items, and between $71 \%$ and $93 \%$ for the 9 FM satisfaction items. Analysis of the 55 available audiotapes produced an average score of 9.38 (SD 1.34; range 611) out of possible score of 11 for intervention content fidelity. Negotiating an agenda for the conversation and offering prognostic information were the intervention components most poorly adhered to (prognostic information not offered in $26 \%$ of cases). Other components were present in $74-98 \%$ of ACPs. A more detailed analysis of ACP content will be reported elsewhere.

Primary outcome: family perception that EoL wishes were discussed and met

Of the 116 dyads with complete primary outcome data, $23 \mathrm{FM}$ (43\%) allocated ACP and 21 FM (33\%) allocated usual care reported that the patient's EoL wishes were discussed and met (difference 10\%; 95\% Cl: -2 to $8, p=0.27$ ). 28 (53\%) FM allocated ACP and 22 (35\%) FM allocated usual care reported that EoL wishes were discussed (difference $18 \%, 95 \% \mathrm{Cl}$ : 0 to $36, p=0.05$ ); and 41 (79\%). FM allocated ACP and 49 (78\%) FM allocated usual care reported that EoL wishes were met (difference 1\%, 95\% Cl: -14 to $16, p=0.89)$ (Table 3$)$. Having an ACD (OR $=3.7(95 \%$ $\mathrm{Cl}: 1.7$ to $8.2, p=0.001)$ and higher satisfaction with care $(\mathrm{OR}=1.2$ for a 1 point increase on a scale from 0 to $24,95 \% \mathrm{Cl}$ : 1.1 to 1.4 , $p=0.004$ ) were significantly associated with FM report of EOL wishes being discussed and met.

Documentation of EoL wishes

Medical record review showed a greater prevalence of: formal ACDs (74\% ACP vs $6 \%$ usual care, $p<0.0001)$; documentation of a Substitute Decision Maker (SDM) (72\% vs $27 \%, p<0.0001$ ); and documentation of preference for Place of Death (POD) (75\% vs $43 \%, p<0.0001)$ in the ACP arm. Rates of discussion of preferences other than in formal ACD were high in both groups (76\% vs $69 \%$, $p=0.29$ ), with no differences in EoL documentation of discussions with the patient regarding preferences for care in the clinic notes, doctors' letters or inpatient notes, or the timing of these discussions. See Table 4.

Among the 85 patients with documented preferences for POD, there was a significant difference between groups in where patients wanted to die $(p=0.04$, see Table 4$)$. This statistical difference was likely determined by the 10 ACP vs 1 usual-care patients whose preferences could not be categorised (e.g. 'I want to die either at home or in palliative care').

Medical care at the EoL

There were no differences between groups in the care received at the EoL. None or very few patients received CPR $(n=0)$, ventilation $(n=0)$ and surgery $(n=1)$. There were no differences in place of death (hospital $18 \%$ vs $19 \%$, home $19 \%$ vs $24 \%$, 
Table 3 Primary and secondary communication outcomes

\begin{tabular}{|c|c|c|c|c|c|}
\hline & $N$ & $\mathrm{ACP} n(\%)$ & Usual care $n(\%)$ & Difference $(95 \% \mathrm{Cl})$ & $p$-value \\
\hline Wishes known and complied with & 116 & $23(43)$ & $21(33)$ & $10 \%(-2,8)$ & 0.27 \\
\hline EOL wishes met & 116 & $41(79)$ & $49(78)$ & $1 \%(-14,16)$ & 0.89 \\
\hline Family satisfied with care ${ }^{a}$ & 115 & $46(87)$ & $53(85)$ & $-1 \%(-14,11)$ & 0.84 \\
\hline Family present during discussions of EOL care with oncologist ${ }^{b}$ & 67 & $20(56)$ & 19(61) & $-6 \%(-29,18)$ & 0.64 \\
\hline Family found discussions useful $^{b}$ & 60 & $20(65)$ & $20(69)$ & $4 \%(-29,19)$ & 0.71 \\
\hline Family rating of the quality of discussions ${ }^{c}$ & 68 & $16(52)$ & $23(62)$ & $-11 \%(-34,13)$ & 0.38 \\
\hline Discussions with pt helpful to make decisions ${ }^{b}$ & 85 & $20(49)$ & $20(45)$ & $3(-18,25)$ & 0.76 \\
\hline \multicolumn{6}{|l|}{ Patient-reported outcomes (post-intervention) } \\
\hline
\end{tabular}

hospice/palliative care $54 \%$ vs $49 \%$, don't know/other $9 \%$ vs $7 \%, p$ $=0.85)$, or receipt of chemotherapy within 4 weeks of death $(21 \%$ vs $14 \% ; p=0.32$ ). There was no evidence of difference in contact with palliative care $(97 \%$ vs $96 \% ; p=0.81)$.

Concordance between documented preferences and medical care at the EoL

Concordance between documented preferences and EoL care received defined as yes (preferences documented and complied with) vs no (preferences documented but not complied with/ preferences not documented) was higher in the ACP arm for POD (49\% vs $26 \%, p=0.003)$; CPR (75\% vs $23 \%, p<0.0001)$; ICU admission ( $28 \%$ vs $11 \%, p=0.008)$ and ventilation $(49 \%$ vs $12 \%, p$ $<0.0001)$. See Table 4. There was no difference in concordance between chemotherapy received in last 4 weeks, surgery, 'other' significant interventions in the last 2 weeks, or other goals of care.

Communication with family members and HCP

More patients allocated ACP than usual care reported communication of EoL care wishes with their oncologist 6 weeks after the intervention: (20 [30\%] vs 12 [16\%], $p=0.04$; nominated FM 49 [73\%] vs 37 [48\%], $p=0.002$; and another health professional, 34 [52\%] vs $16[21 \%], p=0.0001$, see Table 3$)$. There were no differences in prior communication about EoL wishes at baseline (results not shown).

Quality of care

There was no evidence of differences between groups in: patient satisfaction with care $(p=0.38)$; FM satisfaction with care or with death; whether FM reported patient discussion of EoL care with patient's oncologist, or whether FMs found these discussions to be of high quality or useful (see Table 3).

After death outcomes

There was no evidence of differences between groups in FM stress, distress, physical well being before or after death. There was greater improvement in mental well being from baseline to the bereavement interview in the usual-care group, $p=0.006$ (see Table 5).
Results of sensitivity analyses were similar to the primary analyses (see Supplementary file 1).

\section{DISCUSSION}

This ACP intervention increased prevalence of ACDs and documentation of SDM, preferred POD, and wishes regarding specific interventions such as CPR. It also increased communication between patients, oncologists and FMs as reported 6 weeks post-intervention, although FMs reported no difference at bereavement. Contrary to our hypotheses, there were no differences in FM's perception that the patient's wishes for EoL care were met or about the quality of death, or FM or patient satisfaction with care. There were no differences in the medical care patients received at the EoL. Differences between groups in concordance between documented preferences and EoL care received, therefore, were largely driven by differences in documentation only. ${ }^{22}$ There was greater improvement in mental well being from baseline to bereavement in the usual-care group, raising the possibility the intervention adversely affected FMs.

This study documents that while formal ACP did result in higher prevalence of formal ACDs and communication between patient, FM and oncologist immediately following ACP, this does not appear to impact medical care, satisfaction or well being. Similar results have been previously demonstrated in other ACP studies conducted in cancer. Jones et al. ${ }^{13}$ found that an ACP intervention increased discussion with FMs and with health-professionals, but intervention participants were less happy with their communication and satisfaction with healthcare was lower. Wright et $\mathrm{al}^{8}$ found that EoL discussions did not affect rates of major depressive disorder, or 'worry' in patients, and Stein et al. ${ }^{23}$ found no differences in patient knowledge or patient anxiety or depression between groups randomised to receive an ACP intervention. These findings are consistent with previous reviews of the ACP literature, which have concluded the evidence for the impact of ACP on satisfaction with healthcare, decisional conflict, anxiety and depression to be limited or equivocal. ${ }^{10,11}$ This indicates that, while patient and family well -being are often cited as important 
Table 4 Medical record review: Concordance between documented preferences for EOL care and care received at the EoL. Difference, $95 \% \mathrm{Cl}$, and $\mathrm{p}$-values are for Documented and complied with vs (Documented not complied with or not documented)

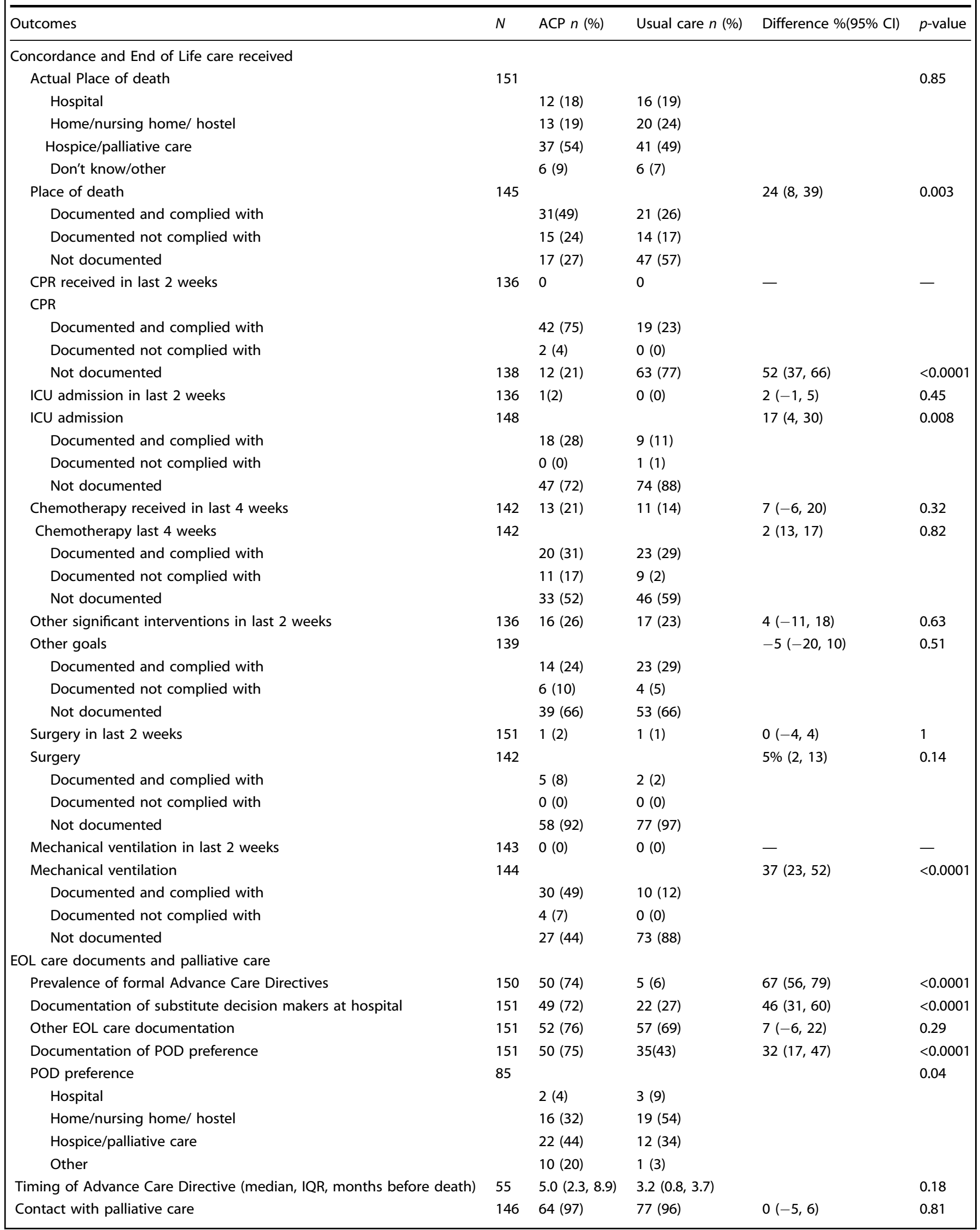


Table 4 continued

\begin{tabular}{|llllll}
\hline Outcomes & $N$ & ACP $n(\%)$ & Usual care $n(\%)$ & Difference \%(95\% Cl) & $p$-value \\
\hline Timing of palliative care (median, IQR, months before death) & 135 & $2.0(.5,6.9)$ & $1.6(0.9,4.3)$ & & 0.68 \\
\hline
\end{tabular}

\begin{tabular}{|c|c|c|c|c|}
\hline \multicolumn{5}{|c|}{ Pre-bereavement (mean overall assessments) ${ }^{a}$} \\
\hline Anxiety (HADS) & 10.2 & 10.4 & $-0.2(-0.6,0.2)$ & 0.49 \\
\hline Depression (HADS) & 8.8 & 8.9 & $-0.1(-0.6,0.4)$ & 0.42 \\
\hline Mental well being (SF-12) & 46.1 & 45.6 & $0.5(-3.8,4.7)$ & 0.83 \\
\hline Anxiety (HADS) & 0.9 & 1.1 & $-0.2(-1.0,0.6)$ & 0.65 \\
\hline Depression (HADS) & -0.7 & -1.2 & $0.5(-0.3,10.3)$ & 0.24 \\
\hline Physical well being (SF-12) & -0.8 & -1.5 & $0.8(-1.6,3.1)$ & 0.52 \\
\hline Mental well being (SF-12) & 2.9 & 9.9 & $-7.0(-12.0,-2.0)$ & 0.006 \\
\hline Impact-of-events (IES) & 23.5 & 23.0 & $0.5(-5.4,6.3)$ & 0.88 \\
\hline
\end{tabular}

goals of ACP, ACP interventions may be of limited benefit in regards to patient or FM well being.

These results are in contrast to two previous studies in cancer that found ACP to increase the frequency of out-of-hospital ${ }^{8,9,23}$ and out-of-ICU care. ${ }^{8}$ In our study, prudent use of aggressive interventions at the EoL, documented refusals of treatment (e.g. DNR forms), and referral to palliative care services all appeared to be routine. In this Australian cancer-specific study, almost no patients (regardless of allocated arm) received aggressive care. No patients received CPR or ventilation, $\sim 80 \%$ died outside of acute hospital and satisfaction with care was high in both groups. Almost all had contact with palliative care and documented discussions with healthcare providers several months prior to death, and FM perception that wishes were met was high (78\% vs $79 \%$ ). ACP interventions may, therefore, have limited impact on medical care in settings where aggressive EoL care is already rare and there is routine access to palliative care.

Three studies outside of the oncology setting have found ACP improves compliance with EoL wishes. ${ }^{12,24,25}$ It is not possible to reliably determine the cause of such difference due to differences in methodology and the intervention. ${ }^{22}$ It is possible that the study intervention failed to increase compliance with EoL wishes in a setting where satisfaction with care was high, and documented discussions of EoL wishes, routine. It is also possible that the study intervention may not have been suitable for the cancer setting. 'Respecting patient Choices' has been widely used and evaluated. $^{12,26}$ However, three quarters of our intervention participants completed ACP, half documented their preferences, and 18 accepted information regarding life expectancy. The mean length of the discussions was $57 \mathrm{~min}$ with a range of $18-120 \mathrm{~min}$. It is likely that the range in length of meeting is dependent upon the patient and/or FMs engagement in the intervention, or possibly on the particular style and skill of the ACP facilitator. Offering life expectancy information was the least reliable measure for intervention standardisation, indicating possible facilitator reluctance, or patient reluctance (patients not wishing to receive this information, or preferring to discuss this with their oncologist) and/or suboptimal timing of the conversations (a median of 5.5 months before death). This may represent intractable problems with ACP (some patients may not wish to engage in $A C P$, the optimal timing of conversations is contested and interventions are difficult to standardise), and suggest further that the physician-patient relationship may play a central role in the quality of EoL care planning. ACP programmes, facilitated by health professionals external to the treating team, therefore may not be ideal. Ongoing development in the field points to the need for an ongoing process of integrated discussion, and our results support this conclusion.

There was greater improvement in mental well being in the usual-care group compared to the intervention group. It is possible that patients who have discussed their wishes become less satisfied with their overall care, if compliance with those wishes is not met. Other ACP studies in cancer have found similar results. ${ }^{27}$ It is also possible that this is a type I error, given the large number of comparisons undertaken.

\section{LIMITATIONS}

This study has important limitations. There was a high proportion of missing primary outcome data, as is common in EoL studies. Although sensitivity analyses did not reveal any obvious bias, results should be interpreted with this in mind. Nearly all participants had contact with palliative care and study questionnaires may have prompted control participants to discuss EoL preferences. Oncologists participating in the study also treated patients in both groups, raising the possibility of contamination of results. Furthermore, it is likely that a referral bias exists. Oncologists may have referred patients most receptive to ACP conversations, and rates of referral to palliative care were high (available data suggests that $\sim 80 \%$ of Australian Cancer patients are admitted to palliative care during their final hospital admission. ${ }^{28}$ Therefore there are inherent sources of bias.

This study also has notable methodological strengths. The study employed a multi-site RCT design, in the absence of any gold standard measures to assess concordance tested reliability of nonvalidated tools and paid attention to protocol adherence through audiotape analyses of the ACP discussions. Rates of drop out and missing data are acceptable for an EoL study ${ }^{29}$ and rigorous sensitivity analyses to test the robustness of results were conducted.

None-the-less, our results raise important questions about the content, structure and efficacy of formal ACP interventions. Further evidence is needed to establish an evidence base for the capacity of ACP to influence the quality of EoL care across 
cultures, and care settings. As future steps, we recommend research should be based on a clear conceptual model of ACP, which considers the role of the patient-doctor relationship. Studies should pay attention to biases, response rates, patient and family reported outcomes and should consider cluster randomised designs to minimise the effect of potential contamination and systemic shifts in care.

\section{CONCLUSION}

We have shown that an ACP intervention for Australian patients with cancer increased communication and documentation of EoL wishes but did not affect their care at the EoL, patient well -being or family well -being. The results of this cancer-specific, randomised trial suggest that ACP interventions have limited benefits in cancer services where aggressive EoL care is rare, and access to palliative care is routine and widespread.

\section{ACKNOWLEDGEMENTS}

We thank Professor Jane Hall for proposing and undertaking an analysis of the inclusion of health care cost estimates in the data collected, and the inclusion of the discrete choice experiment. We also thank Jonathan Cebon for providing clinical leadership in recruitment of patients and protocol development at the Austin Hospital.

\section{AUTHOR CONTRIBUTION}

S.B.J. coordinated development of the study materials and collection of data, contributed to data analysis and interpretation and drafted the manuscript. M.L.B. undertook the data analysis. M.H.N.T. developed the Research Application in collaboration with W.S., K.D., J.M.C., P.N.B. and M.L.B.. J.M.C., W.S. K.D., P.N.B. and B. E.K. refined the research methods and developed the Advanced Care Planning (ACP) intervention for cancer patients. S.C., P.B. and W.S. provided clinical leadership in recruitment of patients and protocol development. L.V. and N.F. contributed to development of study materials and collection of data. M.R.S. and B.E.K. developed the segment of the ACP relating to estimating and communicating patient life expectancy. All authors reviewed and approved the manuscript.

\section{ADDITIONAL INFORMATION}

Supplementary Information is available for this paper at https://doi.org/10.1038/ s41416-018-0303-7.

Ethics approval: Ethics approval for the study was obtained from the: Sydney Local Health District (RPA Zone) Human Research Ethical Committee, Australia (Protocol number X13-0064) and all participating hospitals. Trial Registration: Australia and New Zealand Clinical Trials Registry ACTRN1261300128871.

Conflict of Interest: The authors have completed and submitted the ICMJE Form for Disclosure of Potential Conflicts of Interest. B.E.K. reported receiving personal fees from Roche and Novartis for lectures and advisory board membership, as well as nonfinancial support from Amgen, for work outside of the submitted work.

Funding: This study was funded by The National Health and Medical Research Council (grant number APP 1050596) and is administered through the University of Sydney.

Note: This work is published under the standard license to publish agreement. After 12 months the work will become freely available and the license terms will switch to a Creative Commons Attribution 4.0 International (CC BY 4.0)

\section{REFERENCES}

1. Wright, A. A. et al. Family perspectives on aggressive cancer care near the end of life. JAMA 315, 284-292 (2016).

2. Razmaria, A. A. Chemotherapy use, performance status, and quality of life at the end of life. JAMA 314, 1879 (2015).

3. Higginson, I. J. Research challenges in palliative and end of life care. BMJ Support. Palliat. Care. 6, 3-4 (2016)
4. Lorenz, K. A. et al. Quality measures for symptoms and advance care planning in cancer: a systematic review. J. Clin. Oncol. 24, 4933-4938 (2006).

5. Sudore, R. L. et al. Defining advance care planning for adults: a consensus definition from a multidisciplinary Delphi Panel. J. Pain. Symptom Manag. 53, 821-32. e1 (2017).

6. Narang, A. K., Wright, A. A. \& Nicholas, L. H. Trends in advance care planning in patients with cancer: Results from a national longitudinal survey. JAMA Oncol. 1, 601-608 (2015).

7. Aslakson, R. A. \& Lorenz, K. Being CAREFuL about improving end-of-life care in hospitals. Lancet 390, 97-98 (2017).

8. Wright, A. A. et al. Associations between end-of-life discussions, patient mental health, medical care near death, and caregiver bereavement adjustment. J. Am. Med. Assoc. 300, 1665-1673 (2008).

9. Mack, J. W., Weeks, J. C., Wright, A. A., Block, S. D. \& Prigerson, H. G. End-of-life discussions, goal attainment, and distress at the end of life: predictors and outcomes of receipt of care consistent with preferences. J. Clin. Oncol. 28, 1203-1208 (2010).

10. Brinkman-Stoppelenburg, A., Rietjens, J. A. C. \& van der Heide, A. The effects of advance care planning on end-of-life care: a systematic review. Palliat. Med. 28, 1000-1025 (2014).

11. Houben, C. H., Spruit, M. A., Groenen, M. T., Wouters, E. F. \& Janssen, D. J. Efficacy of advance care planning: a systematic review and meta-analysis. J. Am. Med. Dir. Assoc. 15, 477-489 (2014).

12. Detering, K. M., Hancock, A. D., Reade, M. C. \& Silvester, W. The impact of advance care planning on end of life care in elderly patients: randomised controlled trial. BMJ 340, 847 (2010).

13. Jones, L. et al. Advance care planning in advanced cancer: can it be achieved? An exploratory randomized patient preference trial of a care planning discussion. Palliat. Support. Care. 9, 3-13 (2011).

14. Johnson, S. et al. Advance care planning in patients with incurable cancer: study protocol for a randomised controlled trial. BMJ Open 6, e012387 (2016).

15. Kiely, B. E. et al. Using scenarios to explain life expectancy in advanced cancer: attitudes of people with a cancer experience. Support. Care Cancer 21, 369-376 (2013).

16. Cohen, J. A., Coefficient of Agreement for Nominal Scales. Educational and Psychological Measurement 20, 37-46 (1960).

17. Engelberg, R., Downey, L. \& Curtis, J. R. Psychometric characteristics of a quality of communication questionnaire assessing communication about end-of-life care. J. Palliat. Med. 9, 1086-1098 (2006).

18. Zigmond, A. S. \& Snaith, R. P. The hospital anxiety and depression scale. Acta Psychiatr. Scand. 67, 361-370 (1983).

19. Ware, J. E., Kosinski, M. \& Keller, S. D. A 12-Item Short-Form Health Survey: construction of scales and preliminary tests of reliability and validity. Med. Care 34, 220-233 (1996).

20. Hawthorne, G., Osborne, R. H., Taylor, A. \& Sansoni, J. The SF36 Version 2: critical analyses of population weights, scoring algorithms and population norms. Qual. Life. Res. 16, 661-673 (2007)

21. Bell, M. L. \& Fairclough, D. L. Practical and statistical issues in missing data for longitudinal patient-reported outcomes. Stat. Methods Med. Res. 23, 440-459 (2014).

22. Johnson, S. B., Butow, P. N., Kerridge, I., Bell, M. L. \& Tattersall, M. H. N. How well do current measures assess the impact of advance care planning on concordance between patient preferences for end-of-life care and the care received: a methodological review. J. Pain. Symptom Manag. 55, 480-495 (2018).

23. Stein, R. et al. Randomized controlled trial of a structured intervention to facilitate end-of-life decision making in patients with advanced cancer. J. Clin. Oncol. 31, 3403-3410 (2013).

24. Morrison, R. S. et al. The effect of a social work intervention to enhance advance care planning documentation in the nursing home. J. Am. Geriatr. Soc. 53, 290-294 (2005).

25. Schamp, R. \& Tenkku, L. Managed death in a PACE: pathways in present and advance directives. J. Am. Med. Dir. Assoc. 7, 339-344 (2006).

26. Hammes, B. J., Rooney, B. L. \& Gundrum, J. D. A comparative, retrospective, observational study of the prevalence, availability, and specificity of advance care plans in a county that implemented an advance care planning microsystem. J. Am. Geriatr. Soc. 58, 1249-1255 (2010).

27. Jones, L. et al. Advance care planning in advanced cancer: can it be achieved? An exploratory randomized patient preference trial of a care planning discussion. Palliat. Support. Care. 9, 3-13 (2011).

28. Connolly, A. B. S., Allingham, S., Clapham, S., Quinsey, K. \& Foskett, L. Patient outcomes in palliative care in Australia. National compendium report. (University of Wollongong, Wollongong, 2016).

29. Preston, N. J. et al. Recommendations for managing missing data, attrition and response shift in palliative and end-of-life care research: part of the MORECare research method guidance on statistical issues. Palliat. Med. 27, 899-907 (2013). 